Running Head: COPING STRATEGIES IN COMMUNITY INTEGRATION

\title{
The Enhancement of Community Integration: Coping Strategies of Chinese Parents of Children with Autism Spectrum Disorders
}

\author{
Bernard P. H. Wong
}

Department of Counselling and Psychology, Hong Kong Shue Yan University

\section{Shui-fong Lam}

Department of Psychology, The University of Hong Kong

\section{Doris Leung, Daphne Ho, and Peter Au-Yeung}

Heep Hong Society

Correspond with: Bernard P. H. Wong, Department of Counselling and Psychology, Hong Kong Shue Yan University, 10 Wai Tsui Crescent, Braemar Hill Road, North Point, Hong Kong.

E-mail: phwong@hksyu.edu 


\section{Abstract}

This study presents a collaborative research project by school psychologists and educators in Hong Kong. It investigated the coping strategies used by Chinese parents of children with Autism Spectrum Disorders $(\mathrm{N}=380)$ to enhance their children's community integration and how these strategies were related to their perceptions of community integration and willingness to engage their children in community activities. A factor analysis of questionnaire responses revealed three clusters of coping strategies: Constructive, avoidant, and confrontational strategies. Parents’ willingness to engage their children in community activities was predicted positively by their use of constructive strategies but negatively by their use of avoidant strategies. Confrontational strategies had no predictive power. Parents who used constructive strategies often tended to report more positive emotions and perceive greater importance for engaging their children in community activities.

Keywords: Coping Strategies, Community Integration, Autism Spectrum Disorders, Parental Perception, Hong Kong, Chinese 


\section{Background Information: School Psychology in Hong Kong}

Hong Kong has been a pioneer in the development of school psychology

(called educational psychology in Hong Kong, after the British designation) in Asia (Ding, Kuo, \& Van Dyke, 2008) with the establishment in 1981 of the first school psychology training program in Asia (Hu, Oakland, \& Salili, 1988). Today, around 160 school psychologists currently practice in Hong Kong. School psychologists in Hong Kong are trained with the scientist-practitioner model (Lam, 2006). Their services have gone beyond the traditional role of psychometricians or 'gatekeepers' for special education (Lam \& Mak, 1998). Some of them work in special schools but the majority of them work in mainstream schools, providing school-based services that include assessment, intervention, and consultation (Forlin, 2010). There are also school psychologists engaging in both research and teaching at universities. These school psychologists collaborate closely with the frontline school psychologists and educators to understand the needs of children so as to develop suitable intervention programs. The study reported in this paper is an example of collaboration between school psychologists from universities and educators in the front line to understand the difficulties of the parents who want to integrate their children with Autism Spectrum Disorders (ASD) into the community. The study results shed light on psychological services and intervention strategies that might help children with ASD and their parents in community integration.

\section{Parents of Children with ASD}

Parents of children with psychological and developmental disabilities are at a higher level of stress than those with typically developing children (e.g., Beck, Daley, Hastings, \& Stevenson, 2004). Parents of children with ASD report higher levels of 
parental stress and psychological distress than parents of children with other etiologies—such as intellectual disabilities and physical handicaps (e.g., Sanders \& Morgan, 1997). The prevailing symptoms of ASD include communication and language difficulties, deficiencies in social interactions, social isolation, inappropriate emotional reactions, and repetitive, self-stimulating behaviors (American Psychiatric Association, 2000). These symptoms may pose additional difficulties for parents of children with ASD and could lead to a higher level of distress in parenting and care-giving. However, not all parents of children with ASD report high levels of stress and difficulties. Many parents report positive signs of resilience and adjustment. Parents of children with ASD adjust better when they receive social support (Altiere \& von Kluge, 2008) and are equipped with appropriate coping strategies that assist them through difficult situations (Pottie \& Ingram, 2008). Families may be more functional when they display ample positive coping strategies (Paster, Brandwein, \& Walsh, 2009).

Despite the importance of positive coping strategies, there is little systematic analysis of what 'positive' coping strategies actually are. Only a few studies have been conducted on the types of coping strategies adopted by the parents of children with ASD (Hastings et al., 2005; Pottie \& Ingram, 2008). In addition, little is known about the applications of these findings to Asian parents of children with ASD. As asserted by Theron and Donald (2012), researchers and practitioners shall be aware of the cultural differences as they attempt to translate knowledge reported in Western studies into practice in other cultures. There is a need for Asian school psychologists to find out the extent to which the knowledge generated in the West is valid in their cultures and societies (Daley, 2002). Research on the coping strategies of Asian parents of children with ASD is needed. 


\section{Community Integration}

The coping strategies investigated by the few previous studies were broad based and without focus on any specific context (e.g. Hastings et al., 2005; Paster, Brandwein, \& Walsh, 2009; Pottie \& Ingram, 2008). Coping is context-dependent (Carver, Scheier, \& Weintraub, 1989). Thus, investigations of coping behaviors must go beyond broad based behaviors by considering specific contexts. Community integration is a specific context that deserves attention. Indeed, integration has become an important part of the development of educational policies and practice around the world (Farrell, 2004). Community integration offers a rich mix of learning opportunities to children with disabilities (Dunst, Trivette, Hamby, \& Bruder, 2006). Nevertheless, children with moderate to severe developmental disabilities overwhelmingly participate in non-inclusive recreational and leisure activities (Modell, Rider, \& Menchetti, 1997). The difficulties in coping with the additional demands of physical duties in engaging their children in community activities and the perceived stigmatization from the public (Pelchat, Lefebvre, \& Perreault, 2003) may contribute to lower community participation.

The difficulties experienced by parents of children with ASD in community integration are likely due to the disruptive behaviors associated with ASD (Kraijer, 2000). These difficulties are aggravated further as children with ASD generally are not identifiable by their physical appearances (Sanders \& Morgan, 1997). As a result, parents of children with ASD experience more difficulties obtaining others’ understanding and acceptance of the abnormal behaviors displayed by their children. Many parents of children with ASD report feeling stigmatized (Gray, 2002) and stressed when going out as a family in the community (Higgins, Bailey, \& Pearce 
2005). Therefore, there is a need to examine the effective coping strategies adopted by parents of children with ASD. This study was a collaboration between school psychologists at the University of Hong Kong and educators in the Heep Hong Society, a non-profit social services organization that provides educational services to children with a variety of disabilities in Hong Kong. We investigated the coping strategies that Chinese parents of children with ASD use to cope with community integration, and examine how these strategies are related to parents' perceptions of the importance and difficulties of community integration, and their willingness to engage their children in community activities.

\section{Method}

\section{Participants}

The participants were the parents of 380 Chinese of children with ASD in Hong Kong. Their children attended one of the 22 special preschool centers operated by the Heep Hong Society. The majority (82\%) of the respondents were mothers; fathers made up 17\% while grandparents and other family members made up $1 \%$ of the sample. The majority were between ages 30 and 49 (93\%) years, married (93\%), and about half (45.5\%) held full-time jobs. The ratio between boys and girls with ASD was 9 to 1 . The mean age of these children was 4.5 years with a range from 1.50 to 7.00 years and a standard deviation of .97 .

\section{Procedures}

Parents completed a questionnaire anonymously at home and returned it to the school personnel. Most items in this questionnaire were generated in a pilot study by a focus group comprising six parents of preschool-aged children with ASD, six teachers, 
and six therapists from the Heep Hong Society. The focus group identified community activities that are important to children of ASD, potential difficulties, and coping strategies parents use to deal with these difficulties.

\section{Measures}

Perceived importance of community activities. Parents indicated how important it was for them and their children to participate in 25 community activities (e.g., shopping at supermarket) using a 4-point Likert scale ranging from 1 (not important) to 4 (very important). The 25 community activities were identified by the focus group and could be classified into five domains (Wehman, 2006; i.e., shopping, restaurants, transportation and mobility, health and personal care, and leisure and recreation; see Table 1). The five domain scores were averaged to indicate the overall importance in community activities. The Cronbach's alpha of the five domain scores was .90.

(Insert Table 1 about here)

Perceived difficulty in the participation of community activities. Using a 4-point Likert scale ranging from 1 (not difficult) to 4 (very difficult), the parents indicated the degree of difficulty their children and they experienced when participating in these 25 activities. The five domain scores were averaged to indicate the overall difficulty level in community activities. The Cronbach's alpha of the five domain scores was .87 .

Use of coping strategies. Using a 4-point Likert scale ranging from 1 (never) to 4 (always), parents indicated how often they adopted 24 coping strategies while 
their children participated in community activities. These strategies were also generated by the focus group. A principal-component factor analysis was conducted on the scores of these 24 items. With reference to the results of the Scree Test (Catell, 1978), three factors were extracted. The first, second, and third factors explained $18.90 \%, 9.21 \%$ and $6.89 \%$ of the total variance respectively. To ensure that the items in each factor had distinct and substantial factor loadings, we eliminated five items from further data analyses. Two of these items had factor loadings of less than .35 on any of the three factors and three had factor loadings of more than .35 on more than one factor. The 19 items included in the final analyses are presented in Table 2. An examination of the items and the factor loadings suggests that Factor 1 includes strategies of constructive actions (e.g., seek help and support from professionals), Factor 2 avoidant strategies (e.g., take my child out less often), and Factor 3 confrontational strategies (e.g., lodge complaints to the people and authorities concerned). The item scores in a factor were averaged to indicate the frequency of the use of strategies in that factor.

(Insert Table 2 about here)

Willingness to participate in community activities. Willingness to participate in community activities was measured by four items (e.g., "It is my pleasure to take my child to community activities”). The parents indicated their agreement to these statement using a 4-point Likert scale ranging from 1 (disagree strongly) to 4 (agree strongly). The scores on these four items were averaged to indicate the degree parents were willing to engage their ASD children in community activities. The Cronbach’s alpha of the four scores was .86. 
Emotions evoked during participation in community activities. These emotions were measured by a scale adapted from the Emotional Functioning Scale (Diener, Smith, \& Fujita, 1995). The parents indicated how often they felt 15 emotions (e.g., joy, embarrassment) when their children engaged in community activities using a 7-point scale ranging from 1 (never) through 4 (half and half) to 7 (always). Negative emotion was indicated by the average rating of 12 negative emotions whereas positive emotion was indicated by the average rating of 3 positive emotions. The Cronbach's alphas of the 12 negative emotions and the three positive emotions were .91 and .73 , respectively.

\section{Results}

\section{Perceived Importance and Perceived Difficulty of Community Activities}

Table 1 presents the descriptive statistics of the importance and difficulty of community activities perceived by parents. Parents tended to agree that most of the community activities were important and did not see engaging their child in them as too difficult. The top three important community activities in the parents' perceptions were crossing a road, seeing a physician, and playing in a playground. The top three difficult community activities in the parents’ perception were seeing a dentist, watching a movie in a cinema, and having hair cut at a barber shop.

\section{Willingness and Emotions in Participation in Community Activities}

Table 3 presents the descriptive statistics of the parents' willingness and their positive and negative emotions in engaging their children in community activities. In general, the parents were willing to engage their children in community activities. 
They also experienced more positive than negative emotions when they engaged their children in community activities, $t=18.02, d f=377, p<.001$.

(Insert Table 3 about here)

\section{Coping Strategies}

Table 3 also presents the descriptive statistics for the three clusters of coping strategies. The most frequently used strategies were constructive strategies and the least frequently used were confrontational strategies. The correlations of these three clusters of coping strategies with the other variables are also presented in Table 3.

Perceived importance, perceived difficulty and coping strategies. Parents’ perceived importance of engagement in community activities was associated with their use of constructive strategies. On the other hand, parents' perceived difficulties when engaged in community activities were associated positively with their use of avoidant strategies.

Coping strategies and willingness. To examine whether the three clusters of coping strategies could predict parents' willingness in engaging their children in community activities, a multiple regression was conducted with parents’ willingness regressed on the three clusters of coping strategies. The model explained $17 \%$ of total variance, $F(3,355)=24.00, p<.001$. Parents' willingness to engage their children in community activities was predicted positively by their use of constructive strategies $(\beta=.28, p<.001)$ but negatively by their use of avoidant strategies $(\beta=-.34, p<.001)$. In addition, it was not predicted by their use of confrontational strategies $(\beta=.03, p$ 
$>.05)$.

Coping strategies and emotions. To examine whether the three clusters of coping strategies could predict parents’ positive and negative emotions in engaging their children in community activities, two multiple regressions were conducted. First, parents' positive emotions were regressed on the three clusters of coping strategies. The model explained $10 \%$ of the total variance, $F(3,359)=12.47, \mathrm{p}<.001$. Parents' positive emotions were predicted positively by their use of constructive actions ( $\beta$ $=.20, p<.05)$. However, they were predicted negatively by their use of avoidant strategies $(\beta=-.27, p<.001)$, but not by their use of confrontational strategies $(\beta=.06$, $p>$.05). Second, parents' negative emotions were regressed on the three clusters of coping strategies. The model explained $23 \%$ of the total variance, $F(3,357)=35.86, p$ $<.001$. Parents' negative emotions were predicted positively by their use of avoidant strategies $(\beta=.47, p<.001)$; however, they were not predicted by their use of constructive actions $(\beta=.04, p>.05)$ or confrontational strategies $(\beta=.01, p>.05)$.

\section{Discussion}

The present study has revealed the underlying structure of the coping strategies used by Chinese parents of children with ASD in community integration. Parents' willingness to engage their children in community activities was predicted positively by their use of constructive strategies but negatively by their use of avoidant strategies. In addition, the use of constructive strategies was positively associated with emotions and perceived importance to engage children in community activities.

The present study was conducted in Hong Kong and its findings have 
relevance to Asian countries generally, which have very different cultural backgrounds than Western countries. An interesting contrast between the East and the West is revealed when the items in the three clusters of coping strategies in the present study are compared to those in the Way of Coping Questionnaire (Folkman \& Lazarus, 1988). This questionnaire is used widely in Western countries to measure problem-focused coping and emotional-focused coping of people in stressful situations. Problem-focused coping aims at managing the stressful situation or doing something to alter the source of stress. It includes confrontive coping, planful problem solving, and positive reappraisal. Emotion-focused coping aims to reduce the emotional distress that is associated with the situation. It includes distancing, self-controlling, seeking social support, self-blame, and wishful thinking. Previous studies in the West have found that good adjustment was associated positively with problem-focused coping but negatively with emotion-focused coping (e.g., Hastings, Allen, McDermott, \& Still, 2002; Kim, Greenberg, Seltzer, \& Krauss, 2003).

A close examination of the items in the cluster of constructive strategies in the present study reveals that these findings in the West may not apply to the East. The constructive strategies in the present study included strategies of problem-focused coping (e.g., 'Prepare preventive measures so as to minimize possible difficulties’) as well as strategies of emotional-focused coping (e.g., 'Share feelings with other parents'). Chinese parents did not see problem-focused and emotional-focused coping as two distinct entities. In addition, they did not see confrontation as a part of constructive strategies. In the Way of Coping Questionnaire (Folkman \& Lazarus, 1988), confrontive coping is one of the components in problem-based coping, which is usually associated with better adjustment. However, confrontation was perceived by the Chinese parents in the present study as a distinct entity by itself. Moreover, they 
rarely used it and even when they did, it was not associated with better adjustment as shown in Table 3.

The different findings of the present study and previous studies in the West are understandable given the cultural differences between the East and the West. Chinese culture, like many other collectivistic cultures in Asia, has an emphasis on interdependence and interpersonal harmony. Some emotional-focused strategies, such as seeking support from family, are indeed functional because they are compatible with the cultural norm in China. Confrontational strategies, such as lodging complaints and blaming others for problems, may not be considered as effective problem-solving strategies by Chinese parents because it undermines interpersonal harmony. Some of the emotional-focused strategies in the cluster of constructive strategies in the present study can be seen as relationship-focused strategies, which aim at managing, regulating, and preserving relationships during stressful times. Mak and Ho (2007) argued that relationship-focused coping is more suitable for understanding caregiver perceptions within collectivistic cultures.

Relationship-focused coping may have important implications for services for Asian parents of children with ASD. Luong, Yoder, and Canham (2009) found that many Asian parents of children with ASD considered school as the primary and biggest supportive entity. School psychologists may use school as a platform to organize more self-help groups and mutual support groups for these parents. These interventions provide them with opportunities to share similar care-giving concerns and build up a strong interpersonal support system among them.

This study has provided information about what community activities were perceived as most difficult by Chinese parents of children with ASD. The two most difficult domains were health and personal care, and leisure and recreation. These 
findings have practical implications for school-based psychology services. For instance, school psychologists may develop and implement community-based instructional programs for integration (e.g., Wehman, 2006). These educational programs can target the community activities that are difficult for children with ASD and equip them and their parents with skills and techniques for participation in these community activities. They can help prepare children with ASD for the difficult scenarios that they may encounter through the use of social stories and practice. The teaching package developed by the Heep Hong Society (2009) is an exemplary curriculum in this respect. Drawing on research findings and techniques of behavior modification, the teaching package includes lessons on participation in various community activities. These teaching materials are helpful resources for both educators and parents who want to enhance the integration of children with ASD into the community.

The present study has revealed the underlying structure of the coping strategies adopted by Chinese parents of children with ASD. The findings have practical implications for school psychologists in their development and implementation of psycho-educational programs for this group of children and their families. Despite these contributions, the present study has some obvious limitations. The present study adopted a cross-sectional design. The correlational nature of the data obtained in the present study leave the casual relationships among variables ambiguous. It is impossible to draw a causal linkage between coping strategies, perceived importance and perceived difficulties of community activities. It would be of practical significance for service providers to know the directionality of causality between these variables in order to develop suitable intervention programs for children with ASD. To pursue this line of research and to determine the directionality 
of causality, future studies may consider longitudinal designs so as to allow analyses of time series. Another limitation of the present study was its use of self-reports. Although self-reports are appropriate to measure parents' subjective feelings and thoughts, such as emotions, willingness, and perceived importance, they may not be able to capture the actual behaviors of the parents in engaging their children in community activities. Future studies may consider objective records of participation or third-party reports. 


\section{References}

Altiere, M. J., \& von Kluge, S. (2008). Family functioning and coping behaviors in parents of children with autism. Journal of Child and Family Studies, 18, 83-92.

American Psychiatric Association. (2000). Diagnostic and statistical manual of mental disorders (Revised 4th ed.). Washington, DC: Author.

Beck, A., Daley, D., Hastings, R.P., \& Stevenson, J. (2004). Mothers’ expressed emotion towards children with and without intellectual disabilities. Journal of Intellectual Disability Research, 48, 628-638.

Carver, C. S., Scheier, M. F., \& Weintraub, J. K. (1989). Assessing coping strategies: A theoretically based approach. Journal of Personality and Social Psychology, $56,267-283$.

Cattell, R. B. (1978). The scientific use of factor analysis. New York: Plenum.

Daley, T. C. (2002). The need for cross-cultural research on the pervasive developmental disorders. Transcultural Psychiatry, 39, 531-550.

Diener, E., Smith, H., \& Fujita, F. (1995). The personality structure of affect. Journal of Personality and Social Psychology, 69, 130-141.

Ding, Y., Kuo, Y-L., Van Dyke, D. C. (2008). School psychology in China (PRC), Hong Kong and Taiwan: A cross-regional perspective. School Psychology International, 29, 529-548.

Dunst, C. J., Trivette, C. M., Hamby, D. W., \& Bruder, M. B. (2006). Influences of contrasting natural learning environment experiences on child, parent and family well-being. Journal of Developmental and Physical Disabilities, 18, 235-250.

Farrell, P. (2004). School psychologists: Making inclusion a reality for all. School 
Psychology International, 25, 5-19.

Folkman, S., \& Lazarus, R. S., (1988). Ways of Coping Questionnaire Research edition. Palo Alto, CA: Consulting Psychologists Press.

Forlin, C. (2010). The role of the school psychologist in inclusive education for ensuring quality learning outcomes for all learners. School Psychology International, 31, 617-630.

Gray D. (2002). 'Everybody just freezes. Everybody is just embarrassed’: Felt and enacted stigma among parents of children with high functioning autism. Sociology of Health and Illness, 24, 734-749.

Hastings, R. P., Allen R., McDermott, K. \& Still, D. (2002). Factors related to positive perceptions in mothers of children with intellectual disabilities. Journal of Applied Research in Intellectual Disabilities, 15, 269-275.

Hastings, R. P., Kovshoff, H., Brown, T., Ward, N.J., Espinosa, F. D., \& Remington, B. (2005). Coping strategies in mothers and fathers of preschool and school-age children with autism. Autism, 9, 377-391.

Heep Hong Society. (2009). Shequ shiying le rongrong: zibizheng ertong sheshi jiaocai tao [Training package for the community integration of children with autism syndrome disorders]. Hong Kong: Author.

Hu, S., Oakland, T., \& Salili, F. (1988). School psychology in Hong Kong. School Psychology International, 9, 21-28.

Higgins, D. J., Bailey, S. R., \& Pearce, J. C. (2005). Factors associated with functioning style and coping strategies of families with a child with autism spectrum disorder. Autism, 9, 125-137.

Kim, H. W., Greenberg, J. S., Seltzer, M. M., \& Krauss, M. W. (2003). The role of coping in maintaining the psychological well-being of mothers adults with 
intellectual disability and mental illness. Journal of Intellectual Disability Research 47, 313-327.

Kraijer, D. (2000). Review of adaptive behaviour studies in mentally retarded persons with autism/pervasive developmental disorder. Journal of Autism and Developmental Disorders, 30, 31-47.

Lam, S-F. (2006). Educational psychology in Hong Kong. In S. R. Jimerson, T. Oakland, \& P. Farrell (Eds.), The handbook of international school psychology (pp.147-157). Thousand Oaks, CA: Sage.

Lam, S-F., \& Mak, Y-S. (1998). The changing roles of educational psychologists in Hong Kong. School Psychology International, 19, 317-340.

Luong, J., Yoder, M. K., \& Canham, D. (2009). Southeast Asian parents raising a child with autism: A qualitative investigation of coping styles. The Journal of School Nursing, 25, 222-229.

Mak, W. W. S., \& Ho, G. S. M. (2007). Caregiving perceptions of Chinese mothers of children with intellectual disability in Hong Kong. Journal of Applied Research in Intellectual Disabilities, 20, 145-156.

Modell, S., Rider, R., \& Menchetti, B. (1997). An exploration of the influence of educational placement on the community recreation and leisure patterns of children with developmental disabilities. Perceptual and Motor Skills, 85, 695-704.

Paster, A., Brandwein, D., \& Walsh, J. (2009). A comparison of coping strategies used by parents of children with disabilities and parents of children without disabilities, Research in Developmental Disabilities, 30, 1337-1342.

Pelchat, D., Lefebvre, H., \& Perreault, M. (2003). Differences and similarities between mothers’ and fathers' experiences of parenting a child with a 
disability. Journal of Child Health Care, 7, 231-247.

Pottie, C. G., \& Ingram, K. M. (2008). Daily stress, coping, and well-being in parents of children with autism: A multilevel modelling approach. Journal of Family Psychology, 22, 855-864.

Sanders, J. L., \& Morgan, S. B. (1997). Family stress and adjustment as perceived by parents of children with autism or Down syndrome: Implications for intervention. Child and Family Behavior Therapy, 19, 15-32.

Theron, L. C., \& Donald, D. R. (2012). Educational psychology and resilience in developing contexts: A rejoinder to Toland and Carrigan (2011). School Psychology International. Advance online publication. doi: $10.1177 / 0143034311425579$

Wehman, P. (2006). Life beyond the classroom: Transition strategies for young people with disabilities. Baltimore: Paul H. Brookes. 
Table 1

Means (and Standard Deviations) for the Importance and Difficulty of Community Activities Perceived by Parents

\begin{tabular}{|c|c|c|}
\hline Activity & Importance & Difficulty \\
\hline \multicolumn{3}{|l|}{ Shopping } \\
\hline Shopping at a toy store & $2.54(.94)$ & $1.81(.86)$ \\
\hline Shopping at a super market & $3.14(.87)$ & $1.67(.80)$ \\
\hline Shopping at a wet market & $2.65(1.04)$ & $1.99(1.00)$ \\
\hline Shopping at a shopping mall & $2.91(.96)$ & $1.83(.90)$ \\
\hline Shopping at a shoes store & $2.88(.94)$ & $1.95(.97)$ \\
\hline Shopping in average & $2.82(.69)$ & $1.85(.67)$ \\
\hline \multicolumn{3}{|l|}{ Restaurants } \\
\hline Dinning at a fast food restaurant & $2.96(.92)$ & $1.81(.89)$ \\
\hline Dinning at a Chinese restaurant & $2.95(.89)$ & $1.88(.93)$ \\
\hline Dinning at a café & $3.00(.91)$ & $1.87(.92)$ \\
\hline Restaurants in average & $2.97(.78)$ & $1.85(.80)$ \\
\hline \multicolumn{3}{|l|}{ Transportation and mobility } \\
\hline Crossing a road & $3.68(.67)$ & $1.67(.89)$ \\
\hline Taking a public bus & $3.23(.93)$ & $1.41(.69)$ \\
\hline Taking a taxi & $2.51(1.03)$ & $1.20(.50)$ \\
\hline Riding on metro & $3.14(.94)$ & $1.53(.80)$ \\
\hline Riding on an elevator & $3.16(.97)$ & $1.38(.70)$ \\
\hline
\end{tabular}


Taking a flight on airplane

Riding on an escalator

Transportation and mobility in average

Health and personal care

Seeing a physician

Seeing a dentist

Having hair cut at a barber's shop

Using a public toilet

Health and personal care in average

\section{Leisure and recreation}

Going to a library

Watching a movie in a cinema

Taking photographs at a studio

Playing in a playground

Visiting friends and relatives

Swimming in a beach or public swimming pool

Leisure and recreation in average
$2.42(1.13)$

$2.06(1.11)$

$3.17(.93)$

$1.40(.66)$

$3.06(.67)$

$1.48(.51)$
$3.49(.75) \quad 1.71(.91)$

$3.24(.98)$

$3.00(1.21)$

$3.02(1.02)$

$2.68(1.23)$

$3.36(.90)$

$1.78(.98)$

$3.28(.66)$

$2.17(.82)$

$2.78(.93)$

2.47 (1.07)

$2.17(1.04)$

$2.70(1.12)$

$2.59(.96)$

$2.59(1.13)$

$3.48(.78)$

$1.61(.82)$

$3.11(.91)$

$1.80(.90)$

$3.03(.95)$

$1.84(.94)$

$2.88(.65)$ 
Table 2

Factor Loadings of the Coping Strategies

Coping Strategies
Factor 1

Constructive

Strategies
Factor 2

Avoidant

Strategies

.06

Share feelings with other parents

Enroll my child in community

.55

$-.44$

$-.02$

integration training programs

Seek help and support from

.54

$-.25$

.05

professionals

Prepare preventive measures so as to

.52

.03

$-.35$

minimize possible difficulties

Cultivate good habits in my child

.52

$-.41$

$-.14$

since early days

Take my child out more often so as

to strengthen his/her experience

Talk to myself that my child's

.50

.09

.16

behaviours will be improved

Participate in training activities so as

.49

$-.14$

.20

to equip myself with better skills

Talk to myself that I am able to

.48

$-.01$

.16

handle the problem 
Use some simple skills (e.g., explain

.48

$-.01$

.16

the reasons, be firm, praise my

child)

Seek help and support from family

.46

.00

$-.20$

Leave the scene immediately

.43

.28

$-.29$

Ignore the negative comments from

.42

.28

.00

the public

Take my child out less often

.08

.55

Blaming myself for my inability to

.29

.53

guide and teach my child

Give in to my child and satisfy

.02

.48

$-.33$

his/her demands

Go to places that not many people

.34

.42

$-.15$

visit

Lodge complaints to the people and

.28

$-.05$

.58

authorities concerned

Attribute my child’s problems to the

.05

.30

.40

bias of the public

Note. Loadings above .35 are shown in bold type. 
Table 3

Descriptive Statistics and Correlations of the Variables

\begin{tabular}{|c|c|c|c|c|c|c|c|c|c|}
\hline Variables & Mean & 1 & 2 & 3 & 4 & 5 & 6 & 7 & 8 \\
\hline 1. Willingness & $\begin{array}{l}3.36 \\
(.60)\end{array}$ & -- & & & & & & & \\
\hline 2. Positive & 4.58 & $.39 * *$ & -- & & & & & & \\
\hline Emotion & (1.20) & & & & & & & & \\
\hline 3. Negative & 2.81 & $-.31 * *$ & $-.39 * *$ & -- & & & & & \\
\hline Emotion & (1.09) & & & & & & & & \\
\hline 4. Perceived & 3.01 & $.16^{* *}$ & $.15^{* *}$ & $.13^{*}$ & -- & & & & \\
\hline Importance & (.59) & & & & & & & & \\
\hline 5. Perceived & 1.90 & $-.30 * *$ & $-.37 * *$ & $.44^{* *}$ & .03 & -- & & & \\
\hline Difficulty & $(.56)$ & & & & & & & & \\
\hline 6. Constructive & 2.89 & $.21 * *$ & $.17 * *$ & $.10^{*}$ & $.33 * *$ & .01 & -- & & \\
\hline Strategies & $(.47)$ & & & & & & & & \\
\hline 7. Avoidant & 2.22 & $-.29 * *$ & $-.24 * *$ & $.46^{* *}$ & .02 & $.43 * *$ & $.12 *$ & -- & \\
\hline Strategies & $(.57)$ & & & & & & & & \\
\hline 8. Confrontational & 1.43 & -.01 & .03 & $.14^{* *}$ & .04 & .05 & $.15 *$ & $.24 * *$ & -- \\
\hline Strategies & $(.57)$ & & & & & & & & \\
\hline
\end{tabular}

Note. Numbers in parentheses are standard deviations. ${ }^{*} p<.05,{ }^{* *} p<.01$ 
Bio Statements

Bernard Pak-ho Wong, PhD, is a Senior Lecturer in the Department of Counselling and Psychology at Hong Kong Shue Yan University. He has received professional training in school psychology and is currently working on projects that bridge the gap between theory and practice in school psychology. His research interests include special educational needs, positive psychology, school-based intervention, experiential learning and adventure-based education, and positive youth development. Address: Department of Counselling and Psychology, Hong Kong Shue Yan University, 10 Wai Tsui Crescent, Braemar Hill Road, North Point, Hong Kong. Email: phwong@hksyu.edu

Shui-fong Lam, PhD, is an Associate Professor in the Department of Psychology at the University of Hong Kong where she is the Director of the Master Program in Educational Psychology, a professional training program for school psychologists.. Her research interests lie in achievement motivation, teaching/learning strategies, parenting, and positive psychology. Address: Department of Psychology, The University of Hong Kong, Pokfulam Road, Hong Kong. Email: lamsf@hku.hk Doris Leung, MA (Social Work), was a social worker in the Heep Hong Society, a non-governmental organization that provides early intervention and professional 
support for children with special needs and their families. She is now the superintendent of Precious Blood Children's Village, a charity organization that takes care of children who lack family support. Address: No 1 Bridge, Lung Yeuk Tau, Fanling, N.T., Hong Kong Email: superi@pbcv.org.hk

Daphne Ho, MA (Chinese Linguistics), PGD (Psychology), BSc (Speech \& Hearing Sciences), was a speech therapist in the Heep Hong Society, a non-governmental organization that provides early intervention and professional support for children with special needs and their families. She is now a speech therapist in private practice. Address: 1703A-1705, Landmark North, Sheung Shui, N.T., Hong Kong. Email: daphne@wonderword.com.hk

Peter Au-Yeung, MBA, BSocSc (Social Work), is the Assistant Director in the Heep Hong Society, a non-governmental organization that provides early intervention and professional support for children with special needs and their families. Address: Heep Hong Society, G1, Tung Yu House, Tai Hang Tung Estate, Kowloon. Email: pay@heephong.org 